\title{
Quaderni
}

QUADERNI Communication, technologies, pouvoir

\section{Le forum de discussion : entre activités éditoriales et expertises spontanées}

\section{Éric Sotto}

\section{(2) OpenEdition \\ 1 Journals}

\section{Édition électronique}

URL : http://journals.openedition.org/quaderni/1070

DOI : 10.4000/quaderni.1070

ISSN : 2105-2956

Éditeur

Les éditions de la Maison des sciences de l'Homme

\section{Édition imprimée}

Date de publication : 5 mai 2017

Pagination : 23-31

Référence électronique

Éric Sotto, "Le forum de discussion : entre activités éditoriales et expertises spontanées », Quaderni [En ligne], 93 | Printemps 2017, mis en ligne le 05 mai 2019, consulté le 02 janvier 2020. URL : http:// journals.openedition.org/quaderni/1070; DOI : 10.4000/quaderni.1070 


\section{$D$ ossier}

\section{Le forum de discussion : entre activités éditoriales et expertises spontanées}

\section{Éric Sotto}

Novancia Business School Paris DICEN-CNAM

(Dispositifs d'Information et de Communication à l'Ère Numérique)
Nous interrogeons les pratiques éditoriales et conversationnelles observées dans un forum envisagé comme espace documentaire numérique, avec pour objectif de mieux saisir la manière dont les producteurs de contenus, capables de nouvelles activités cognitives (Monnoyer-Smith, 2011) ${ }^{1}$ s'inscrivent dans une activité éditoriale cadrée. Nous appuyons notre propos sur les résultats obtenus d'une analyse manuelle, interactionniste et conversationnelle d'un corpus de messages publiés dans le forum de discussion Booknode qui réunit des lecteurs-usagers. Cette analyse est complétée par un comptage manuel des contributeurs et des messages postés. Sans généraliser, nous appréhendons le forum comme un espace hybride se caractérisant par l'émergence de nouvelles formes d'expression et de construction de connaissances, sans l'intervention de professionnels ou d'experts de l'objet de discussion. Le forum constitue un espace documentaire coopératif autogéré par une myriade d'auteurs éclairés et quelques éditeurs assurant une fonction de régulateur pour favoriser la construction d'un document pérenne. L'expression d'une opinion individuelle est le registre de prise de parole dominant, se traduisant par un empilement de points de vue argumentés et d'expériences commentées, constituant une nouvelle forme d'expertise profane.

\section{Le forum : une plateforme sociotechnique à dominante documentaire}

Nous ne reviendrons pas sur l'abondante littérature traitant des forums de discussion. De façon générale, ces travaux interrogent la notion de genre et se focalisent sur l'expression des affects (Marcoccia, 2011) $)^{2}$ ou s'inscrivent dans une 
perspective managériale centrée sur les communautés virtuelles $\left(\right.$ Cova, 2006) ${ }^{3}$. En définitive, les spécificités de l'activité éditoriale restent encore à découvrir. Nous appréhendons le forum de discussion comme une plateforme sociotechnique collaborative de co-production à dominante documentaire. En effet, dans un forum, les participants sont motivés par la production collective de contenus (s'entraider et expérimenter) constituant ainsi un document numérique. Pour compléter, le forum est un document inachevé faisant l'objet d'une rédaction collective permanente par des participants/membres en réseau (Zacklad, 2007). Dans un tel cadre, les participants sont amenés à prendre en charge la totalité de l'activité éditoriale afin d'assurer la cohérence et la compréhension du document en cours de construction. Notre objet d'étude porte sur un forum public de discussion : https://booknode.com/forum ${ }_{2}$ une des rares plateformes non marchandes à proposer des conversations publiques, en langue française, sur le support livre numérique et son contenu. Il est édité par la société Media Node SAS créée

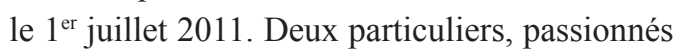
des livres, identifiés par leurs pseudos Virgile et Miracl sont à l'origine de ce projet. Les créateurs affichent clairement leurs objectifs : se démarquer des sites marchands de livres et constituer une base de connaissance littéraire en s'appuyant sur l'aide et l'expérience des particuliers. Nous posons l'hypothèse que sur cette plateforme sociotechnique, les participants organisent et régulent leurs échanges, sans l'intervention de tiers, aboutissant à la production d'un savoir profane. Dans une approche empirique, nous décrivons le processus interactionnel à l'œuvre, afin de repérer le type d'activité éditoriale qui s'opère ici puis d'identifier de nouvelles formes de production collective de connaissances. Notre échantillon totalise 353 messages déposés par 265 participants uniques - pendant la période du 20 juillet 2011 au 30 janvier 2013 - issus de deux fils de discussion avec une question de départ, à l'initiative d'un participant, incitant les lecteurs à donner un avis général sur le livre numérique comme support physique. Nous inscrivons notre recherche dans le champ de l'interactionnisme et la pragma-linguistique, en empruntant les concepts des interactions quotidiennes défendus par Erving Goffman $(1973,1974)^{5}$ : « face » et « territoire de l'individu », auxquels nous associons des notions issues de l'interaction verbale, développées par Catherine Kerbrat-Orecchioni $(1990,1992,1994)^{6}:$ " relation interpersonnelle », " procédés accompagnateurs », " actes complimenteurs ». De plus, nous exploitons le modèle d'observation d'un dispositif sociotechnique de Josiane Jouët et Coralie Le Caroff $(2013)^{7}$, avec une focale sur leur grille d'observation de la participation (p. 157) qui suggère une série d'observables pour qualifier les propos et repérer des modalités d'engagement comme le registre de prises de parole ou les ressources argumentatives.

\section{Une myriade d'auteurs indépendants assurant l'activité éditoriale}

Dans le forum, trois catégories de participants apparaissent et s'expriment : les deux administrateurs, les six modérateurs et les innombrables contributeurs (21 2676 en 2016). La possibilité d'accéder directement à l'index des sujets de discussion - via Google en saisissant des mots clés évidents comme « livre numérique forum » - sans passer par l'index général, la faible visi- 
bilité des informations relatives aux règles de fonctionnement et aux mentions légales, ainsi que l'affichage de trop brèves informations sur la nétiquette suggèrent une absence de contrôle. Pour prolonger, nous constatons que les six modérateurs ne jouent pas le rôle attendu. Effectivement, un seul message est déposé par un des modérateurs pour livrer une opinion personnelle sur le sujet en cours. Ces acteurs semblent se comporter en participants, sans exercer une fonction de vigiles ou de filtreurs. Pourtant, ils sont détenteurs d'une autorité puisqu'ils peuvent supprimer les contributions et enregistrer les signalements portant sur un contenu offensant ou illégal. De plus, aucune indication ne permet d'identifier clairement un modérateur : absence d'indicateur de statut et de rôle dans la zone conversationnelle, pseudo peu explicite. En évitant toute affirmation, ces derniers ne souhaitent pas exercer un contrôle ou une autorité sur les activités en cours.

Cette forme d'encadrement autorise un accès à tous les publics, sans distinction, et dispense le participant des rituels de politesse/salutation trop appuyés liés à l'ouverture et à la clôture d'une rencontre. Par conséquent, l'entrée en conversation est directe et abrupte, sans véritable séquences préliminaires puisque $7 \%$ des participants utilisent un acte de politesse d'ouverture. La quasi-totalité des interactants s'affranchit des rituels de prise de contact et s'engagent d'emblée dans une relation de nature familière : émoticône ${ }^{8}$ de clin d'œil/sourire/rire, tutoiement, adressage par le pseudo, expressions relâchées, fautes d'orthographe, etc. Ainsi, les statuts, qualités et compétences des participants n'entrent pas dans le jeu d'une interaction qui se veut franche et directe, avec une relation interpersonnelle basée sur l'égalité de compétence s'appuyant sur des relations de proximité. Certes, nous relevons la présence de grades hiérarchiques - automatiquement attribués par le dispositif éditorial en fonction de l'activité des inscrits - mais ces derniers se révèlent trop nombreux et peu différenciés pour justifier d'une réelle autorité. Par exemple, nous distinguons mal les différences entre des « booknodes-gardiens-templierschevaliers-maîtres-vénérables-sacrés ». Ces grades - non mentionnés dans les messages publiés - ne reposent pas sur une réelle compétence ou connaissance. Pour compléter, nous soulignons l'absence de « citations emblématiques » (Lucas, 2009) ${ }^{9}$, plus précisément l'ajout d'aphorismes ou de maximes qui participent à l'affirmation identitaire et à l'appartenance communautaire. Nous en déduisons que le forum ne constitue pas une communauté de lecteurs, mais plutôt un groupe d'utilisateurs avancés désireux de s'exprimer sur le livre numérique. Nous soulignons l'usage massif de modalisateurs - un procédé accompagnateur de type adoucisseur ${ }^{10}$ - qui rendent le propos plus nuancé, moins catégorique, moins affirmatif. Ainsi, les contributeurs n'entendent pas imposer leur point de vue sur les autres membres. Ces procédés traduisent un doute ("je ne pense pas que... »; " ...après c'est mon avis »), montrent une prise en compte de l'opinion des autres («...je veux bien le reconnaître aisément [E]»; « certes, mais est-ce que... »), remettent en cause un jugement (« ... Surtout dites moi si je me trompe »; "je suis d'avis partagé... »). Nous notons l'usage régulier de maximisateurs - un procédé accompagnateur de type intensifieur ${ }^{l l}$ - destinés à mettre en valeur l'ego des participants ou à se montrer reconnaissant envers eux, en remerciant le plus 
souvent chaleureusement (« merci à tous pour vos super avis »; "c'est plutôt sympa ici »), en approuvant les propos ou en montrant un intérêt pour les publications déposées («je suis totalement d'accord... »; "c'est le meilleur argument...»). Enfin, nous soulignons la présence de minimisateurs - un procédé accompagnateur de type adoucisseur - mobilisés ici pour éviter une incursion sur le « territoire de l'individu ${ }^{12}$ » (Goffman, 1973), en indiquant le désir de respecter l'intimité de l'autre, de ménager son égo, de ne pas le contraindre : « ...un petit comparatif si possible merci. »; «j'ai une petite question... ».

Les administrateurs limitent leurs prérogatives et affranchissent les participants de conditions d'accès ou d'utilisation contraignantes. Ils fournissent le cadre général de l'édition éditoriale, en proposant un espace de discussion s'articulant autour de nombreux thèmes et sous-thèmes de discussion imposés mais associés à des sujets de conversation ${ }^{13}$ proposés par les participants, en leur laissant le champ libre pour organiser et réguler leurs échanges. Justement, nous remarquons une seconde activité à la marge, d'autorégulation des échanges, sans la présence active des modérateurs, ce qui montre une forme d'auto-gouvernance du forum sans l'intervention de tiers institutionnels.

\section{Des éditeurs pour éviter l'altération du document ?}

Nous soulignons l'émergence spontanée d'une catégorie d'auteurs participant activement à la régulation et à la structuration de l'échange. Cette activité est d'ailleurs implicitement sollicitée par les administrateurs de la plateforme : «Les contri- butions des utilisateurs ne font l'objet d'aucune modération a priori. Aucun message ne sera donc lu avant sa publication ». Ces contributeurs que nous nommons éditeurs pour les distinguer des modérateurs et pour mettre en évidence leur rôle actif, interviennent pour éviter l'altération du document en cours de construction (abandon du forum, dispersion et éparpillement thématique), plus particulièrement quand celui-ci est menacé dans sa compréhension (définition, explication, synthèse, correction) et dans sa pertinence (recentrage sur le sujet principal, demande d'échange cohérent, ouverture thématique). Les éditeurs ne souhaitent pas exercer une activité de contrôle sur les activités en cours, car leurs propos insistants et réitérés sont nuancés par des actes flatteurs grâce aux procédés accompagnateurs identifiables par des signaux non verbaux et des émoticônes de type adoucisseurs (sourire, rire, clin d'œil), ou par des procédés verbaux désarmeurs ${ }^{14}$, afin de ménager la « face ${ }^{15}$ » des récepteurs (Goffman, 1974) : « ...Ce n'est pas une attaque contre toi, mais toujours dire la même chose... c'est usant [E]». Quatre situations se présentent : Intervention spontanée d'un éditeur pour réguler l'échange / l'intervention est accueillie avec bienveillance par les participants / l'éditeur réagit avec insistance et emphase mais sans réel autoritarisme / l'éditeur n'impose pas son expertise. Pour illustrer notre point de vue, nous proposons de détailler une séquence conversationnelle avec un éditeur pseudo-nommé Milathea.

Un éditeur intervient spontanément pour synthétiser les premiers échanges en vue d'étoffer le débat :

Milathea a écrit : "Ce débat a déjà été ouvert... Il en a résulté la chose suivante: ceux qui sont 
contre la liseuse resteront contre de toute façon... ceux qui possèdent une liseuse vous diront que les deux sont complémentaires et que l'un ne se fera pas sans l'autre... »

L'intervention de l'éditeur est accueillie avec bienveillance : émoticône du sourire, actes verbaux d'opposition sous une forme nuancée :

NiNeGorma a écrit : "Pas toujours, pour moi la liseuse n'est pas complémentaire avec le livre papier et se suffit à elle seule [E] »

L'éditeur réagit, plusieurs fois, avec insistance et emphase, mais sans réel autoritarisme (émoticône de l'embarras, actes verbaux non agressifs), pour éviter les répétitions et pour réitérer sa demande de synthèse des échanges :

Milathea a écrit : «La liseuse n'ABIME PAS les yeux car ce n'est PAS un écran d'ordinateur ! ... Cen'est pas une attaque contre toi, mais toujours dire la même chose.... c'est usant [E]»

L'éditeur n'impose pas son expertise (marqueur d'apaisement). Il met en avant les compétences et connaissances nécessaires aux participants pour déposer des contributions :

Milathea a écrit : "J'aimerais savoir si tous ceux qui répondent "papier» ont déjà essayé une liseuse et peuvent donc répondre avec cohérence à ce débat en toute connaissance de cause. [E]»

Nous soulignons le rôle de l'éditeur Milathea. Ici, un participant prend une initiative, car il estime qu'un risque d'altération du document est possible. Son action est motivée par le désir d'aider à la construction d'une connaissance collective plutôt que sur un rappel des normes comportementales attendues dans l'échange. $\mathrm{Ce}$ travail collaboratif sur un document en construction, nous le retrouvons dans le « Document pour l'Action »(Zacklad, 2003) $)^{16}$. Pour expliquer, à partir d'un message initial, une production sémiotique prend forme et fait l'objet d'une rédaction et modification collective ininterrompue et décentralisée grâce aux interactants qui disposent de prérogatives différentes. Nous constatons que l'enrichissement et le prolongement de la production sémiotique sont surtout assurés par les auteurs-lecteurs, grâce à une «stratégie de documentarisation » (Zacklad, 2007) qui s'appuie sur l'utilisation de techniques annotatives. Autrement dit, des formes graphiques d'ajouts visant à attirer l'attention sur un élément clé de la contribution ou à faciliter la compréhension de l'échange. Par exemple, la citation manuelle est utilisée pour assurer la persistance conversationnelle. En effet, grâce à elle, les interactants ne sont pas obligés de réagir au message précédent ni de respecter exactement le thème principal, ils peuvent prolonger l'échange et assurer sa cohérence en se focalisant sur les messages «à proximité » dans le fil. Autrement dit, sans remonter jusqu'au début de la conversation, mais sans se limiter aux seules dernières contributions. Un bémol, le rôle de l'éditeur ne semble ni déterminant ni indispensable dans la régulation et la nature de l'échange. Des exemples issus du corpus montrent que le locuteur peut se comporter comme un éditeur en déplorant la pauvreté du débat, en regrettant l'absence de construction commune ( je vois des accumulations d'opinons...»; « on tourne séverement en rond...»; « pourquoi rester 
planté sur ce débat... ») mais ces actes coopératifs montrent surtout la bonne volonté de quelques membres pour maintenir une cohérence et un intérêt thématique dans la conversation en cours. Parfois, ces interventions donnent lieu à des réponses de confirmation assimilables aux « actes complimenteurs ${ }^{17} \gg$ (Kerbrat-Orecchioni, 1994) comme les marques d'intérêt destinés à valoriser un auteur : "...C'est vrai que c'est usant ...»; « Je rejoins NiNeGorma et Yaagi. .... Ce débat commence à être gonflant et inutile...»; « Je suis complètement d'accord avec toi! ».

En définitive, dans notre forum, nous repérons une myriade d'auteurs indépendants et peu engagés produisant des contenus bruts et prenant en charge une grande partie de l'activité éditoriale. Cette activité éditoriale est soutenue par des éditeurs pour éviter l'altération du document, plus particulièrement quand celui-ci est menacé dans sa compréhension (explication et synthèse, etc.) et dans sa pertinence (recentrage et enrichissement thématiques, etc.). Le principal intérêt de leurs interventions réside dans la proposition d'une norme de construction commune du document pour éviter les répétitions, ou pour suggérer de lire les messages avant de les poster, etc. Au-delà de l'activité éditoriale, nous portons maintenant un intérêt à une forme d'expertise qui apparaît ici.

\section{Un empilement d'opinions éclairées}

L'activité dominante porte sur un échange de connaissances pratiques et profanes sur le livre numérique. Dans le premier fil, les participants sont sollicités sous la forme de questions pour apporter des réponses, afin de compléter les informations du demandeur : " voila mon problème, je me demande si les livres numériques valent le coup. Est-ce le même plaisir de lire sur une tablette? et surtout est-ce que les livres sont moins chers? ... enfin bref si vous pouviez m'éclairer ce serait super sympa. Merci ». Dans le second fil, la question incitative est plus laconique : "Préférez-vous lire sur une liseuse ou lire un livre papier? Dites-moi ce que vous en pensez [E] ». L'analyse des 353 contributions montre que l'expression d'une opinion individuelle est le registre de prise de parole dominant. Nous interprétons l'opinion individuelle comme une réponse affective, raisonnée ou idéologique à une question de type «que pensez-vous de...? 》 (Eensoo-Ramdani et al., 2011 ; Grivel, 2011) ${ }^{18}$. L'expression de cette opinion met en évidence la prédominance d'un jugement (69\%) basé sur un raisonnement avec des arguments solides (Charaudeau et Maingueneau, 2002) ${ }^{19}$. Dans une moindre mesure cette opinion prend la forme d'une appréciation (27\%). Autrement dit, d'une réaction affective ou d'un point de vue subjectif sans arguments convaincants ${ }^{20}:$ «J'aime sentir les pages entre mes doigts... »; «... je l'adore... c'est vraiment pratique ». Nous relevons une quantité négligeable d'opinions relevant d'une croyance (4\%), plus explicitement d'une assertion, d'une vérité définitive et radicale, bref, d'une « certitude dans l'ignorance » (Jacques, 1985) ${ }^{21}$ : "c'est inadmissible d'avoir inventé ça !...»; «...À bas les tablettes et livres numériques !!! [E]». L'expression d'un point de vue et le témoignage sont les ressources argumentatives mobilisées par les participants. Toutefois, le témoignage sous la forme d'un retour détaillé d'une expérience est la ressource argumentative la plus représentative du forum (38\%). Les participants semblent pri- 
vilégier l'expérimentation à la connaissance, les découvertes et trouvailles aux modes d'emploi et aux notices proposés par les professionnels. D'ailleurs, l'information, plus précisément une connaissance provenant d'une source web extérieure est une ressource argumentative faiblement exploitée (2\%). Certes, nous relevons quelques rares liens vers des sources d'information, mais aucune référence aux experts et professionnels du domaine, dont on semble se méfier ( «ça n'a pas empêché ces paranos d'éditeurs de mettre des DRM [E] ». Nous décelons, tout au plus, quelques références aux proches du locuteur (« je crois que mon cousin...»; « ma meilleure amie... ») mais jamais de références explicites aux acteurs institutionnels du sujet de discussion. En définitive, nous remarquons majoritairement un empilement d'opinions élaborées de profanes « jouant à l'expert » (Besnier et al., 2015) $)^{22}$ sur une thématique principale.

\section{Conclusion}

Dans cet article nous avons restitué les pratiques éditoriales observées dans un forum public de discussion réunissant des discutants sur l'objet physique livre numérique. Nos analyses montrent que les participants organisent et régulent leurs échanges, sans l'intervention de tiers, débouchant sur la production d'une nouvelle forme d'expertise. L'activité éditoriale est assurée par des contributeurs bienveillants s'autorisant des comportements de proximité et soutenue par des éditeurs intervenant spontanément pour éviter l'altération du document en cours de construction. L'activité dominante repose sur l'expression argumentée d'un point de vue individuel sur le livre numérique ou le retour commenté et détaillé d'une expérience personnelle de l'objet. Cet empilement d'opinions de profanes aboutit à une nouvelle forme d'expertise, susceptible de s'inscrire dans un dispositif de co-innovation entre une organisation et ses usagers. Pour nous, le forum constitue un vivier d'observation de micro-usages ordinaires potentiellement exploitables par les organisations. En effet, ce dispositif sociotechnique regroupe des lecteurs-usagers capables de livrer un jugement argumenté, le plus souvent à partir d'expériences personnelles de consommation portant sur des pratiques éphémères, temporaires et banales comme tourner les pages d'un livre assis sur un canapé ou le ranger rapidement dans un sac en marchant. Une nouvelle piste pour les collaborateurs chargés de l'activité innovante dans les organisations... 
$\mathrm{N} \cdot \mathrm{O} \cdot \mathrm{T} \cdot \mathrm{E} \cdot \mathrm{S}$

1. L. Monnoyer-Smith, " La participation en ligne, révélateur d'une évolution des pratiques politiques?», in Participations, $\mathrm{n}^{\circ}$ 1, Paris, 2011, pp. 156-185.

2. M. Marcoccia, «Pourquoi s’intéresser aux forums de discussion? », in Les forums de discussion : agoras $d u$ XXI ${ }^{e}$ siècle ?, Paris, L'Harmattan, 2011, pp. 277-286.

3. B. Cova, « Développer une communauté de marque autour d'un produit de base : L'exemple de my nutella The Community ", in Décisions Marketing, ${ }^{\circ} 42$, Avril-Juin 2006, pp.53-62.

4. M. Zacklad, «Annotation : attention, association, contribution », in P. Salembier, M. Zacklad (eds), Annotations dans les Documents pour l'Action, Paris, Lavoisier, 2007, pp. 29-46.

5. E. Goffman, La mise en scène de la vie quotidienne.

1. La présentation de soi, Paris, Les Éditions de Minuit, 1973. / E. Goffman, La mise en scène de la vie quotidienne. 2. Les relations en public, Paris, Les Éditions de Minuit, 1973. / E. Goffman, Les rites d'interaction, Paris, Les Éditions de Minuit, 1974.

6. C. Kerbrat-Orecchioni, Les interactions verbales, tome 1, Paris, Armand Colin, 1990. / C. KerbratOrecchioni, Les interactions verbales, tome 2, Paris, Armand Colin, 1992. / C. Kerbrat-Orecchioni, Les interactions verbales, tome 3. Variations culturelles et échanges rituels, Paris, Armand Colin, 1994.

7. J. Jouët, C. Le Caroff, L'observation ethnographique en ligne, Paris, Armand Colin, 2013.

8. Pour des raisons de mise en forme, dans les exemples de message les émoticônes sont remplacées par la lettre [E].

9. N. Lucas, «Citation interactionnelle et citation emblématique dans les forums de discussion en ligne », in Communications du IV Ci-dit Colloque international, Nice, 11-13 juin 2009.
10. Un adoucisseur atténue une parole menaçante en contextualisant l'énoncé.

11. Un intensifieur renforce un acte de langage.

12. L'individu dispose de droits qui s'exercent sur un territoire, plus exactement dans un espace physique autour de lui.

13. < Discussions Littéraires < Livres numériques, électroniques, ebooks $<$ «Préférez-vous lire sur liseuse ou livre papier? », "Que pensez-vous des livres numériques et des tablettes?»

14. Un désarmeur tente de désamorcer une éventuelle réaction négative du lecteur compte tenu du caractère brutal de l'énoncé.

15. Ménager l'ego d'un individu, en faisant preuve de tact et de politesse

16. M. Zacklad, « Transactions communicationnelles symboliques et communauté d'action : réflexions préliminaires », in Colloque de Cerisy, Connaissance, Organisation, Activité, Cerisy-la-Salle, 11-18 septembre 2003, <sic 00001122>.

17. Les « actes complimenteurs » sont destinés à valoriser et encourager un individu.

18. E. Eensoo-Ramdani, E. Bourrion, M. Slodzian, M. Valette, « De la fouille de données à la fabrique de l'opinion ", in Les cahiers du Numérique, vol 7, $\mathrm{n}^{\circ}$ 2, Octobre 2011, pp. 15-30. / L. Grivel, « Analyse d'opinions sur internet», in Les cahiers du Numérique, vol 7, n² 2, Octobre 2011, pp. 9-14.

19. P. Charaudeau, D. Maingueneau, Dictionnaire d'analyse du discours, Paris, Seuil, 2002.

20. Ibid.

21. F. Jacques, L'espace logique de l'interlocution, Paris, Puf, 1985.

22. A. Besnier, «L'évaluation sur Internet : une démocratie de l'expertise ? ", in J. Bouchard, E. Candel, H. Cardy, G. Gomez-Méjia (éd.), $L a$ médiation de l'évaluation, Berne, Peter Lang, 2015, pp. 43-60. 
$R \cdot E \cdot S \cdot U \cdot M \cdot E ́$

Dans cet article nous interrogeons les pratiques éditoriales et les expertises en construction dans un forum de discussion. Nous soutenons que le forum constitue un espace documentaire coopératif autogéré par une myriade d'auteurs éclairés et quelques éditeurs assurant une fonction de régulateur. L'expression d'une opinion individuelle est le registre de prise de parole dominant, se traduisant par un empilement de points de vue argumentés et d'expériences commentées, constituant une nouvelle forme d'expertise.

\begin{abstract}
In this paper, we question the editorial practices and the new expertises that are being built in a public discussion forum. We support that the forum constitutes a cooperative documentary domain which is self-administered and self-regulated by a myriad of amateurs and some editors acting as regulators. The expression of an individual opinion is the main modality of intervention, giving a stack of supported points of view and commented experiences which constitute a new form of expertise.
\end{abstract}


